This item was submitted to Loughborough's Research Repository by the author.

Items in Figshare are protected by copyright, with all rights reserved, unless otherwise indicated.

\title{
Investigating vehicle roadway usage patterns on the Shanghai urban expressway system and their impacts on traffic safety
}

\section{PLEASE CITE THE PUBLISHED VERSION}

https://doi.org/10.1080/15568318.2020.1722869

\section{PUBLISHER}

Taylor \& Francis

\section{VERSION}

AM (Accepted Manuscript)

\section{PUBLISHER STATEMENT}

This is an Accepted Manuscript of an article published by Taylor \& Francis in International Journal of Sustainable Transportation on 3 February 2020, available online:

http://www.tandfonline.com/10.1080/15568318.2020.1722869.

\section{LICENCE}

CC BY-NC-ND 4.0

\section{REPOSITORY RECORD}

Yu, Rongjie, Yiyun Wang, Mohammed Quddus, Jian Li, Xuesong Wang, and Ye Tian. 2020. "Investigating Vehicle Roadway Usage Patterns on the Shanghai Urban Expressway System and Their Impacts on Traffic Safety". Loughborough University. https://hdl.handle.net/2134/11877702.v1. 
1 Investigating Vehicle Roadway Usage patterns on the Shanghai

2 Urban Expressway System and Their Impact on Traffic Safety

3

4

5

6

7

8

Rongjie $\mathbf{Y u}^{\mathrm{a}, \mathrm{b}}$, Associate Professor

E-mail: yurongjie@tongji.edu.cn

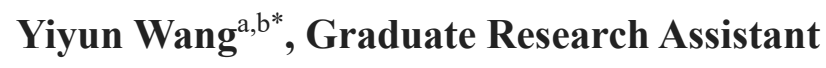

* Corresponding Author

E-mail: 1731347@tongji.edu.cn

Mohammed Quddus , Professor

E-mail: M.A.Quddus@lboro.ac.uk

Jian Li ${ }^{\mathrm{a}, \mathrm{b}}$, Ph.D., Associate Professor

E-mail: tongji_lijian@163.com

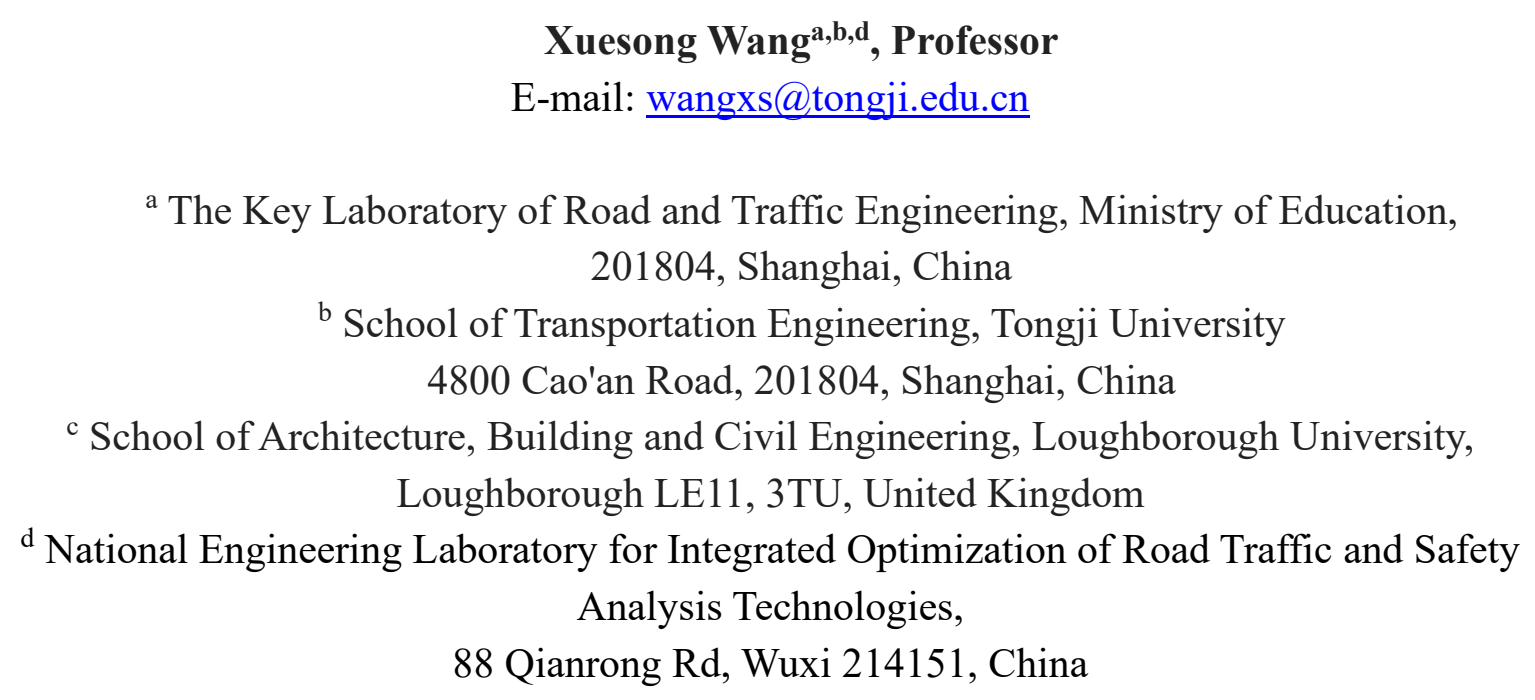




\section{ABSTRACT}

2 Driving behavior has been identified as the most critical factor for crash occurrence. The

3 turbulent traffic flows, attributed by mixed vehicle types and heterogeneous driving 4 behaviors, are main reasons for crash occurrence. Previous studies have investigated the 5 impacts of traffic flow compositions on safety from the aspects of vehicle type (e.g. trucks, 6 passenger cars, motorcycles) and vehicle territory (e.g. local/non-local vehicles). Little 7 research considers how vehicle road use patterns (based on vehicle traveling rates on a 8 roadway system) affect traffic safety and this is the focus of this study. The empirical 9 analysis was conducted based on data from the Shanghai urban expressway system. First, 10 the license plate recognition (LPR) data were utilized to classify the vehicles and identify 11 road use patterns among them using latent class cluster analysis (LCCA). Then, random12 effects hurdle Poisson (REHP) and hurdle negative binominal (REHNB) models were 13 developed with hourly based crash frequency data. The hourly data were used in order to 14 capture the time varying feature of vehicle road use patterns. While considering the 15 excessive zero crash counts and unobserved heterogeneity, random-effects models were 16 employed. From the modeling results it indicated that higher proportions of vehicles with 17 the low-level road use pattern would substantially enhance the possibility of crash 18 occurrence. While proportions of vehicles with the medium-high-level road use pattern had 19 negative impact on crash occurrence likelihood. Finally, the implications for safety 20 improvement from the education and management aspects were discussed.

23 Keywords: Vehicle traveling rate; Vehicle road use pattern; Hourly based crash frequency 24 analysis; Latent class cluster analysis; Random-effects hurdle model 


\section{INTRODUCTION}

2 Traffic safety has become one of the most important issues in transportation. More than

312.5 million people worldwide die each year due to traffic crashes. It is widely 4 acknowledged that more than $90 \%$ of traffic crashes are attributed to human factors (1).

5 The heterogeneous attitudes toward driving and risky behaviors such as violations, 6 aggressiveness or inattention to the roadway environment are all related to crash 7 occurrence. The various driving behaviors are mainly attributed to the drivers' personal 8 characteristics (e.g. driving experience, age and gender), and their familiarity with the 9 roadway infrastructure and operational conditions (1).

11 Since traffic flow is composed with a diverse range of drivers, roadway segments with the 12 same level of traffic volume and road geometry are, therefore, experienced with a varying 13 level of traffic safety. One common consequence is that under the same level of service 14 (LOS), more crashes occurred on holidays in comparison to workdays. A case for this 15 phenomenon is presented in Figure 1, which compares the hourly crash frequency 16 distribution for national holidays and workdays with different traffic volumes on the 17 Shanghai urban expressway system. Under the same volume interval, the mean crash 18 frequency for the roadway network during holidays is about 20 times higher compared to 19 workdays. In regard to this phenomenon, traffic engineers often argue that there are more 20 tourist drivers traveling on holidays, who demonstrate a lack of driving experience for both 21 road and traffic conditions. Therefore, roadway segments with a larger proportion of these 22 drivers are more likely to incur a higher percentage of crashes compared to roadway 23 segments with that of commuting drivers. However, this kind of hypothesis has never been 24 examined and validated with empirical data.

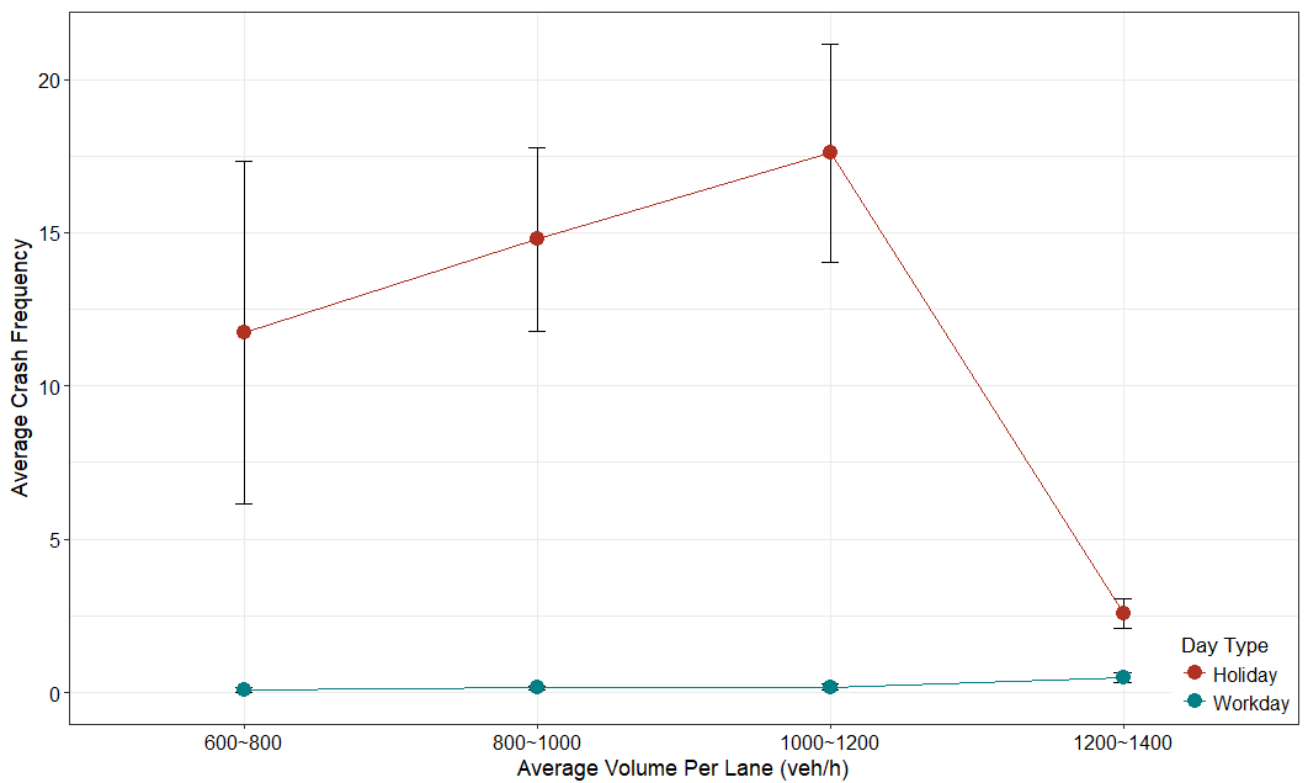


1 In this paper, we use a terminology - road use pattern of vehicles and this is described as 2 whether vehicles sporadically or regularly travel on a roadway system, i.e. spatial and 3 temporal characteristics of traveling rates of vehicles on a roadway system. Figure 2 shows 4 the perspective of present traffic component-related studies and how the focus of this study 5 differs.

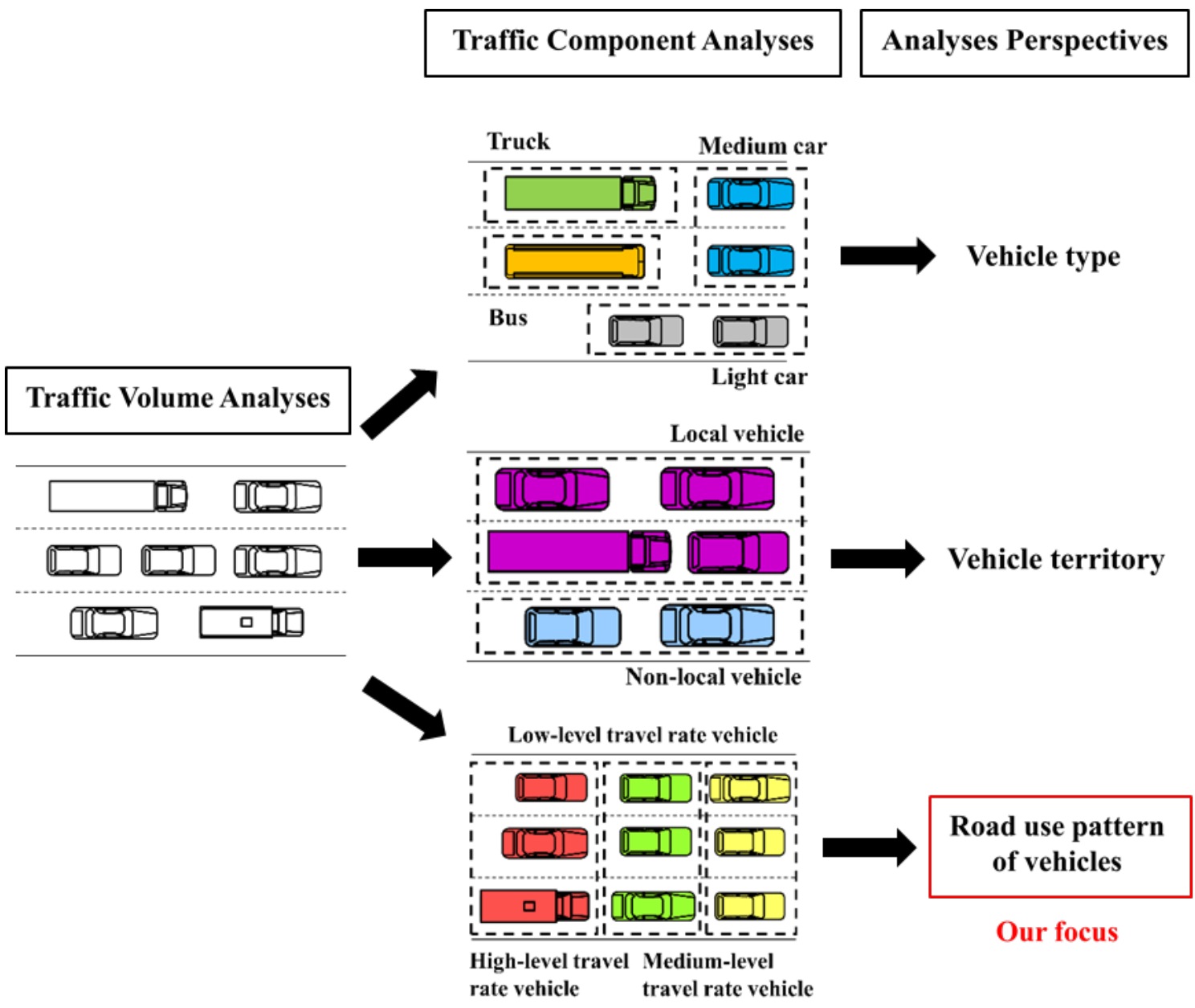

FIGURE 2 Present Traffic Component-Related Studies

10 As for vehicle road use pattern analysis, it aims at understanding motorized travel features

11 and their influencing factors. Vehicles were classified by travel purpose (commercial, 12 recreation and business [2]) and travel frequency (low, medium, and high-level traveling 13 rates [3]). The results of this kind of analysis are critical to traffic planning, operation and 14 management policy. For instance, it will help to: (i) understand a vehicle road use pattern's 15 relationship with urban characteristics (4) and residential environment (5), (ii) evaluate the 16 effect of vehicle road use pattern on traffic flow (6), and (iii) analyze the policies to control 17 urban traffic congestion ( 6 ). 
2 This unprecedented study aims to link vehicle road use patterns on a roadway system with 3 traffic safety through an hourly based crash frequency analysis. The empirical analysis was 4 conducted based on data from the Shanghai urban expressway system. First, the LPR data were utilized to classify vehicles (according to their traveling rates) with the latent class cluster analysis (LCCA) technique. The heterogeneous road use patterns of different vehicles that travel on the Shanghai urban expressway system were identified. In order to avoid important information loss (7) and erroneous parameter estimates (8) during the data aggregation procedure, hourly based crash frequency data were formulated to capture the time varying flow components $(9,10)$. Finally, accounting for the substantial zero crash counts and unobserved heterogeneity, random-effects hurdle Poisson (REHP) and hurdle negative binomial (REHNB) models were applied.

\section{LITERATURE REVIEW}

This section focuses on the methods employed to identify different vehicle road use patterns based on vehicle traveling rates on a roadway system and the crash frequency analysis models adopted to investigate the relationship between vehicle road use patterns and traffic safety.

\section{Vehicle Traveling Rates Analyses}

To identify the heterogeneity among vehicle road use patterns based on traveling rates, both equal width method and clustering method have been applied. For instance, Li et al., (3) proposed an equal width method, taking 0.33 and 0.67 as cut-off points for traveling rate to split vehicles into low, medium, and high-level categories. However, without looking into data characteristics, the equal width method may lead to biased clusters. Cluster analysis, an unsupervised learning technique, was used to divide a heterogeneous dataset into homogeneous subgroups or clusters (11). Guo et al., (2) employed K-means clustering method and identified three sub-groups, which have travel characteristics of commuting vehicles (with high-level traveling rate on workdays), business vehicles (with low-level traveling rate on all types of days), and recreation vehicles (with high-level traveling rate on holidays and low-level on workdays). However, K-means clustering method could be affected by outliers (12), and the statistical properties of this distance-based clustering algorithm are unknown $(13,14)$, thus it is hard to decide the optimal number of clustering groups.

Whereas the statistical properties of probability-based clustering technique known as latent class cluster analysis (LCCA) are better understood $(15,16)$. The LCCA technique has been frequently adopted in transportation studies to deal with the traffic accident heterogeneity issue $(14,17,18)$ and describe the unobserved heterogeneity of drivers $(3)$. 
1 In addition, the LCCA model outperforms K-means clustering method since LCCA can

2 analyze relationships in categorical data and the data used in the analysis do not need to

3 have the same scale or have equal variances (19). Additionally, the model can be

4 statistically tested to identify the best number of classes (20). In this study, the LCCA model

5 is therefore applied to identify the heterogeneity road use patterns among the vehicles

6 traveling on the roadway system.

\section{Hourly Based Crash Frequency Analyses}

9 For hourly based crash frequency analyses, there are several issues that need to be discussed. First, the rare event of crash observations would cause excess zeros within the dataset. In the literature, zero-inflated models have been widely adopted to deal with the excessive zeros $(21,22)$. The model is developed based on the assumption that the presence of extra zeros may come from two states: a true-zero state (where the zones are inherently safe) and a non-zero state (which happen to record zero crashes in an observation period) (23). However, intrinsically safe roadway segments would not exist in reality, which makes this fundamental assumption logically fallacious $(24,25)$. Recently, hurdle models were investigated to be employed as an alternative analysis approach (26). As a two-part model, the first part of the hurdle model is used to decide whether the count value is zero or positive. Given the value is positive, the second part is applied as a count distribution (27). This assumption, where the existence of the zero record was decided by a 'hurdle', is more realistic for traffic crash frequency analysis (28). Hojun et al. (29) utilized a hurdle model to identify the dangerous locations and their contributing factors. Chen et al. (30) applied a hurdle model to investigate the real-time characteristics influencing the traffic safety.

If observations are repeated by roadway segment, and if there are unidentified factors at the segment level (often referred to as the unobserved heterogeneity), then the underlying statistical model has to control for unobserved components in panel data. This would, otherwise, lead to biased parameter estimations and incorrect inferences (31). To deal with this issue, individual roadway segment-based random effects are usually introduced to the model, capturing the unobserved heterogeneity factors for each specific roadway segment (32). Chin (33) developed a random-effects model to examine traffic accident occurrence at signalized intersections. Chen et al. (34) applied a site-specific random-effects model to investigate the real-time environment's impact on crash occurrence. It has been proved that the utilization of random-effects models enhance the model goodness-of-fits $(35,36)$. In this study, considering the excessive-zeros and unobserved heterogeneity issues for hourly based crash frequency analysis data, the site-specific REHP and REHNB models were established to analyze factors influencing crash likelihood and crash frequency. 


\section{DATA}

2 This study utilized the Shanghai urban expressway system as the study area. The study 3 time period was set on October 2012, considering the data availability from different

4 resources period. The data included 7 national holidays (National Day and Mid-Autumn

5 Festival), 6 weekends, and 18 workdays. A total of three datasets were employed: (i) LPR

6 data; (ii) traffic crash data; and (iii) roadway segment geometric data. LPR data were

7 utilized to extract vehicle traveling rate variables and traffic operational status variables,

8 combined with geometric data and crash data, to form the hourly crash frequency analysis

9 data.

\section{License Plate Recognition Data}

12 For the Shanghai urban expressway license plate recognition system, 268 detector points

13 along the system were employed to detect and record the information for each vehicle.

14 Table 1 lists the recorded parameters and the descriptions.

\section{TABLE 1 Description of LPR Data}

\begin{tabular}{ll}
\hline Parameters & Descriptions \\
\hline TIME & Time when a vehicle passes through the detector point \\
TRANSECT ID & ID number of detector points \\
LANE NO & Number of lanes of the segment on which a vehicle is traveling \\
VEHICLE ID & License plate number of vehicles \\
COLOR & License plate color of vehicles: \\
& 01-yellow: large vehicle \\
& 02-blue: light vehicle \\
& 06- black: foreign enterprise or embassy vehicle \\
& 25-white: special vehicle \\
VEHICLE REGION & Region of vehicles \\
SPEED & Vehicle speed: $\mathrm{km} / \mathrm{h}$ \\
\hline
\end{tabular}

17 After the data cleansing process, more than 3 million individual light vehicles were 18 detected along the expressway system. Three features were introduced to describe the 19 vehicle traveling rate characteristics:

(i) Workday Traveling Rate (vehicle's traveling rate on workdays) $=\frac{n_{w o}}{N_{w o}}$

$$
\text { (ii) Weekend Traveling Rate (vehicle's traveling rate on weekends) }=\frac{n_{w e}}{N_{\text {we }}}
$$

$$
\text { (iii) Holiday Traveling Rate (vehicle's traveling rate on holidays) }=\frac{n_{h o}}{N_{h o}}
$$

23 Where $n_{w o}, n_{w e}$ and $n_{h o}$ is the number of days that specific vehicle has traveling record 24 on workday, weekend and holiday. $N_{w o}, N_{w e}$ and $N_{h o}$ equals to 18,6 and 7 respectively 
1 meaning the total number of days for workday, weekend and holiday.

3 The summary statistics and distribution illustrations for vehicle traveling rate features are 4 shown in Table 2.

TABLE 2 Summary Statistics for Vehicle Traveling Rate Features

\begin{tabular}{llllll}
\hline Traveling Rate Features & Mean & s.d. & $\mathbf{1 5}^{\text {th }}$ Percentile & $\mathbf{5 0}^{\text {th }}$ Percentile & $\mathbf{8 5}^{\text {th }}$ Percentile \\
\hline Workday Traveling Rate & 0.34 & 0.32 & 0.06 & 0.24 & 0.82 \\
Weekend Traveling Rate & 0.32 & 0.30 & 0 & 0.17 & 0.67 \\
Holiday Traveling Rate & 0.28 & 0.28 & 0 & 0.14 & 0.57 \\
\hline
\end{tabular}

6

8

In order to develop the LCCA model, the continuous traveling rate feature parameters were discretized into categories. A percentile classification method was used here, which employed the $15^{\text {th }}$ percentile, $50^{\text {th }}$ percentile, and $85^{\text {th }}$ percentile as cut-off. The categories under each traveling rate feature parameters were marked as levels 1-4 corresponding to the degree of traveling rate from low to high. The final classified categories are shown in Table 3. The obtained 12 categories will be used as variables in LCCA model to find heterogeneous road use patterns among vehicles.

TABLE 3 Categories under Each Traveling Rate Feature Parameter

\begin{tabular}{lllll}
\hline Traveling Rate Feature Parameters & Categories & Values & Count & Proportion \\
\hline Workday Traveling Rate & L1 & $<6 \%$ & 820980 & $26 \%$ \\
& L2 & $6 \sim 24 \%$ & 887398 & $28 \%$ \\
& L3 & $24 \% \sim 82 \%$ & 1070361 & $34 \%$ \\
& L4 & $>82 \%$ & 395981 & $12 \%$ \\
\hline Weekend Traveling Rate & L1 & 0 & 917184 & $29 \%$ \\
& L2 & $0 \sim 17 \%$ & 713908 & $22 \%$ \\
& L3 & $17 \% \sim 67 \%$ & 1164131 & $37 \%$ \\
& L4 & $>67 \%$ & 379497 & $12 \%$ \\
\hline Holiday Traveling Rate & L1 & 0 & 916241 & $29 \%$ \\
& L2 & $0 \sim 14 \%$ & 721632 & $23 \%$ \\
& L3 & $14 \% \sim 57 \%$ & 1137273 & $36 \%$ \\
& L4 & $>57 \%$ & 399574 & $12 \%$ \\
\hline
\end{tabular}

\section{Crash Frequency Analysis Data}

18 For crash data, Figure 3 shows the hourly based temporal distribution of crash frequency 19 on the entire expressway system. It demonstrates that the number of crashes reported 20 during 23:00 to 06:00 was limited. The small magnitude of crashes would have minimal 21 contribution to the statistical model and the potential non-reporting event could be included 22 in the model, which therefore might produce biased estimation. Thus the time period of the 
1 final dataset was set from 06:00 to 23:00. A similar time data processing approach can be 2 seen in Mo et al.'s research (37).

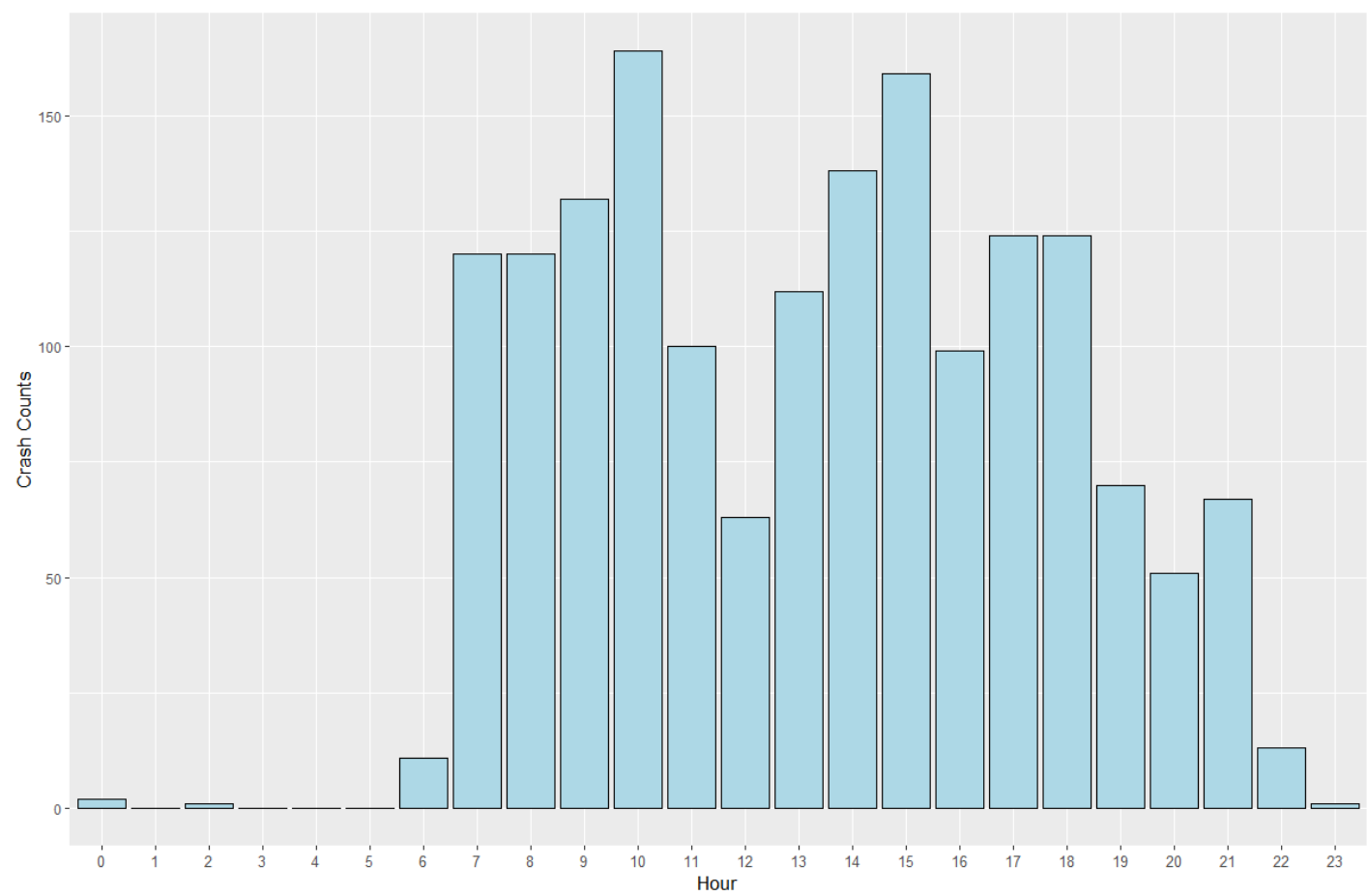

FIGURE 3 Hourly Distribution of Crash Frequency

7 A total of 1,659 crashes which occurred on the corresponding road segments were 8 considered in the analysis. Crash data were aggregated into 1 hour interval data by 191 9 roadway segments, which were split using on-ramps and off-ramps as dividing points (20).

10 After the data aggregation procedure, crash frequency per hour has the mean of 0.0176 per 11 roadway segment and the standard deviation of 0.1666 , with the $98.63 \%$ of the roadway 12 segments possess zero crash count.

14 Geometric characteristics data for each roadway segment were obtained from on-line 15 street-view maps. The summary statistics for geometric variables are shown in Table 4, 16 with the ramp combination types indicated in Figure 4.

17

TABLE 4 Description of Geometric Variables

\begin{tabular}{lll}
\hline Variable & Description & Summary Statistics \\
\hline Length & Roadway segment length $(\mathrm{m})$ & Mean: 923.9 \\
& & s.d.: 536.64 \\
Ramp Type & Ramp combination type: & $1: 64$ \\
& 1. Off-ramp and On-ramp & $2: 36$ \\
\hline
\end{tabular}




\begin{tabular}{|c|c|c|}
\hline & 2. Off-ramp and Off-ramp & $3: 22$ \\
\hline & 3. On-ramp and Off-ramp & 4: 69 \\
\hline & 4. On-ramp and On-ramp & \\
\hline \multirow[t]{3}{*}{ Curve } & If it is a curve section: & 1: 92 \\
\hline & 1. Curve section & 0: 99 \\
\hline & 0. Straight section & \\
\hline \multirow[t]{4}{*}{ Speed Limit Sign } & If there is speed limit sign within the roadway & 1: 65 \\
\hline & segment: & $0: 126$ \\
\hline & 1. Has speed limit sign & \\
\hline & 0. No speed limit sign & \\
\hline \multirow[t]{2}{*}{ Transition Length } & The ratio of transition length of a roadway segment & Mean: 182.5 \\
\hline & (m) & s.d.: 175.04 \\
\hline \multirow[t]{3}{*}{ Speed Limit Value } & The level of speed limit value: & $1: 78$ \\
\hline & 1. $50 \mathrm{~km} / \mathrm{h}$ and $60 \mathrm{~km} / \mathrm{h}$ & 2: 113 \\
\hline & $2.80 \mathrm{~km} / \mathrm{h}$ & \\
\hline \multirow[t]{3}{*}{ DMS } & If there is DMS within the roadway segment: & 1: 57 \\
\hline & 1. Has DMS & $0: 134$ \\
\hline & 0. No DMS & \\
\hline \multirow[t]{4}{*}{ Main Lane } & The level of lane numbers for the main road: & 1: 61 \\
\hline & 1. Has 2 lanes & 2: 84 \\
\hline & 2. Has 3 lanes & 3: 46 \\
\hline & 3. Has 4 lanes and above & \\
\hline \multirow[t]{3}{*}{ Access Lane 1} & The level of lane numbers for the up-access road: & $1: 26$ \\
\hline & 1. Has 1 lane & 2: 165 \\
\hline & 2. Has 2 lanes and above & \\
\hline \multirow[t]{3}{*}{ Access Lane 2} & The level of lane numbers for the down-access road: & $1: 26$ \\
\hline & 1. Has 1 lane & 2: 165 \\
\hline & 2. Has 2 lanes and above & \\
\hline
\end{tabular}
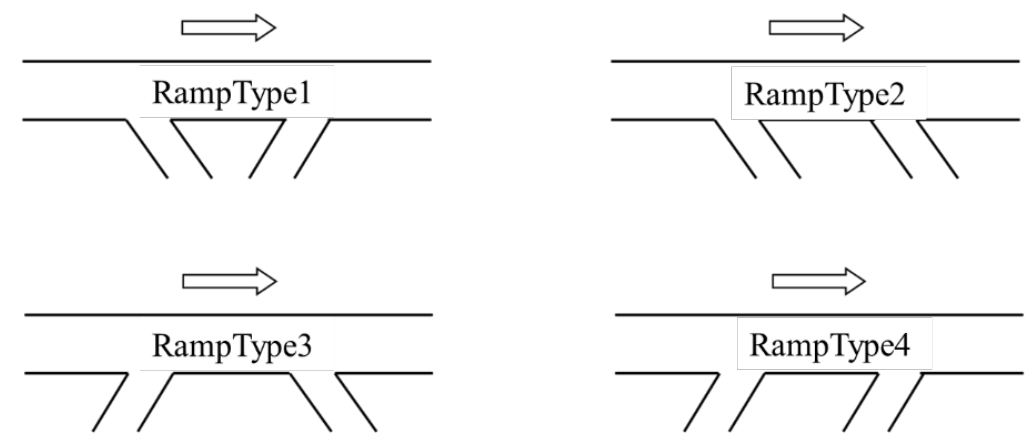

FIGURE 4 Ramp Type Illustration 
1 It is worth mentioning that since no prior assumptions were used about the functional

2 relationships between traffic operational parameters and crash frequency for the Shanghai

3 urban expressway system, different functional forms were tested for the traffic volume and

4 speed variables. This includes: volume and its quadratic form, vehicle miles travelled;

5 speed and its quadratic form, and speed gap (speed limit value minus average speed). In

6 the final analysis results, only the significant variables and the best functional forms were

7 kept.

\section{METHODOLOGY}

\section{Latent Class Cluster Analysis}

12 The LCCA is a probability-based cluster analysis method (38). Like all cluster analysis 13 methods, the LCCA separates the heterogeneous population into groups so that the 14 similarity within the group is maximized and similarity between group elements is minimized (39). Different statistical criteria are available in LCCA output, which can be used to identify the appropriate number of clusters (17). In this study, the LCCA models were developed through a R package named poLCA (40).

Given the set of vehicle-level data, there are $n_{c}$ latent classes to be estimated based on a set of $\mathbf{M}$ categorical vehicle traveling rate feature parameters $(\mathrm{M}=3$ in this study, which are workday traveling rate, weekend traveling rate, and holiday traveling rate). Let the vector $Y_{i}=\left(Y_{i 1}, \ldots, Y_{i M}\right)$ denote the individual vehicle $i$ 's responses to the $\mathrm{M}$ items, where the $Y_{i M}$ takes possible values $1, \ldots, r_{M}$. Let $L_{i}=1,2, \ldots, n_{c}$ be the latent class membership of observation $i$; and let $\mathrm{I}(\mathrm{y}=k)$ be the indicator function, which equals 1 if $y$ equals $k$, and 0 otherwise. Then the contribution by observation $\mathrm{i}$ can be expressed as:

$$
\mathrm{P}\left(Y_{i}=y\right)=\sum_{l=1}^{n_{c}} \gamma_{l} \prod_{m=1}^{M} \prod_{k=1}^{r_{m}} \rho_{m k \mid l}^{I\left(y_{m}=k\right)}
$$

where $\gamma$ is the prevalence or class-membership probability, and $\rho$ is the item-response

To determine the number of clusters, Akaike Information Criteria (AIC), Bayesian Information Criteria (BIC) and Consistent Akaike Information Criteria (CAIC) are usually utilized. Percentage reductions in criteria between competing models were analyzed to determine the classification number (18).

\section{Random Effects Hurdle Count Models}

In this study, the REHP model and REHNB model were developed. Among which, $Y_{i t}$ represents the crash frequency with its observed value $y_{i t}$ for roadway segment $i$ at time interval $t$. The conditional probabilities of responses for the random effects are given by: 


$$
p\left(y_{i t} ; \boldsymbol{b}_{i}\right)= \begin{cases}p_{i t}^{c}\left(b_{i 1}\right) & \text { if } y_{i t}=0 \\ \left(1-p_{i t}^{c}\right) \frac{g_{i}\left(y_{i t} ; \lambda_{i t}^{c}\left(b_{i 2}\right)\right)}{1-g_{i}\left(0 ; \lambda_{i t}^{c}\left(b_{i 2}\right)\right)} & \text { if } y_{i t}>0\end{cases}
$$

$2 \operatorname{logit} p_{i t}^{c}\left(b_{i 1}\right)=\Delta_{i t 1}+z_{i t 1}^{T} b_{i 1}, p_{i t}^{c}$ is the probability of the $i^{t h}$ roadway segment to be in 3 the zero state at time interval $t$.

4 For Poisson distribution:

$$
g_{i}\left(y_{i t} ; \lambda_{i t}^{c}\left(\boldsymbol{b}_{i 2}\right)\right)=\frac{\exp \left(-\lambda_{i t}^{c}\left(b_{i 2}\right)\right) \lambda_{i t}^{c}\left(b_{i 2}\right)^{y_{i t}}}{y_{i t} !}
$$

6 and for negative binomial distribution:

$$
g_{i}\left(y_{i t} ; \lambda_{i t}^{c}\left(\boldsymbol{b}_{i 2}\right)\right)=\frac{\Gamma\left(v^{-1}+y_{i t}\right)}{\Gamma\left(v^{-1}\right) y_{i t} !}\left(\frac{1}{1+v \lambda_{i t}^{c}\left(b_{i 2}\right)}\right)^{v^{-1}}\left(\frac{v \lambda_{i t}^{c}\left(b_{i 2}\right)}{1+v \lambda_{i t}^{c}\left(b_{i 2}\right)}\right)
$$

$8 \operatorname{logit} \lambda_{i t}^{c}\left(b_{i 2}\right)=\Delta_{i t 2}+z_{i t 2}^{T} b_{i 2}, \lambda_{i t}^{c}$ is the expected number of crashes on the $i^{\text {th }}$ roadway 9 segment at time interval $t . \Delta_{i t 1}$ and $\Delta_{i t 2}$ are connector functions corresponding to the $10 \boldsymbol{b}_{\boldsymbol{i} 1}$ and $\boldsymbol{b}_{\boldsymbol{i} 2}$ with their regressors $z_{i t 1}^{T}$ and $z_{i t 2}^{T}$, respectively.

$11 \boldsymbol{b}_{\boldsymbol{i}}$ is the random-effects vector for the $i^{\text {th }}$ roadway segment, and it obeys distribution:

$$
\boldsymbol{b}_{i}=\left(\begin{array}{l}
\boldsymbol{b}_{i 1} \\
\boldsymbol{b}_{i 2}
\end{array}\right) \sim N(\mathbf{0}, \boldsymbol{\Sigma})
$$

13 where $\boldsymbol{b}_{\boldsymbol{i} 1}$ and $\boldsymbol{b}_{\boldsymbol{i} 2}$ are random vectors of the zero part and the positive count part. They 14 are assumed to be independent to each other. Thus, the variance-covariance matrix for them 15 is given as:

$$
\Sigma=\left(\begin{array}{cc}
d_{1}^{2} & 0 \\
0 & d_{2}^{2}
\end{array}\right)
$$

The models were built with NLMIXED procedure in SAS (41). For the model goodnessof-fit evaluations, both Akaike Information Criteria (AIC) and Bayesian Information Criteria (BIC) values were adopted. The AIC is defined as:

$$
A I C=-2 l+2 p
$$

22 and $\mathrm{BIC}$ is defined as:

$$
B I C=-2 l+p \ln (n)
$$

where $l$ is $\log$-likelihood, $p$ is the number of parameters and $n$ is the number of samples. The smaller AIC or BIC value indicates a better fitted model.

\section{MODELING RESULTS}

\section{Vehicle Road Use Patterns Identification}

To identify the optimal number of homogeneous classes representing vehicle traveling rates on the Shanghai urban expressway system, LCCA models with 1-class to 7-class clusters 
1 were estimated. Figure 5 shows the evolutions of BIC, AIC and CAIC for the 7 models. As

2 the number of clusters increases from 1-class to 3-class, the values of BIC, AIC and CAIC

3 reduces substantially. However, the BIC, AIC and CAIC values hardly show additional

4 improvement over the 4 clusters. Thus, the model with 4 clusters was selected, ensuring

5 the classification interpretability and maintaining the model succinct $(14,42)$. The

6 probabilities for 4 clusters under different traveling rate characteristics are shown in Table

75 and the characterizations for each cluster are given in Table 6 .

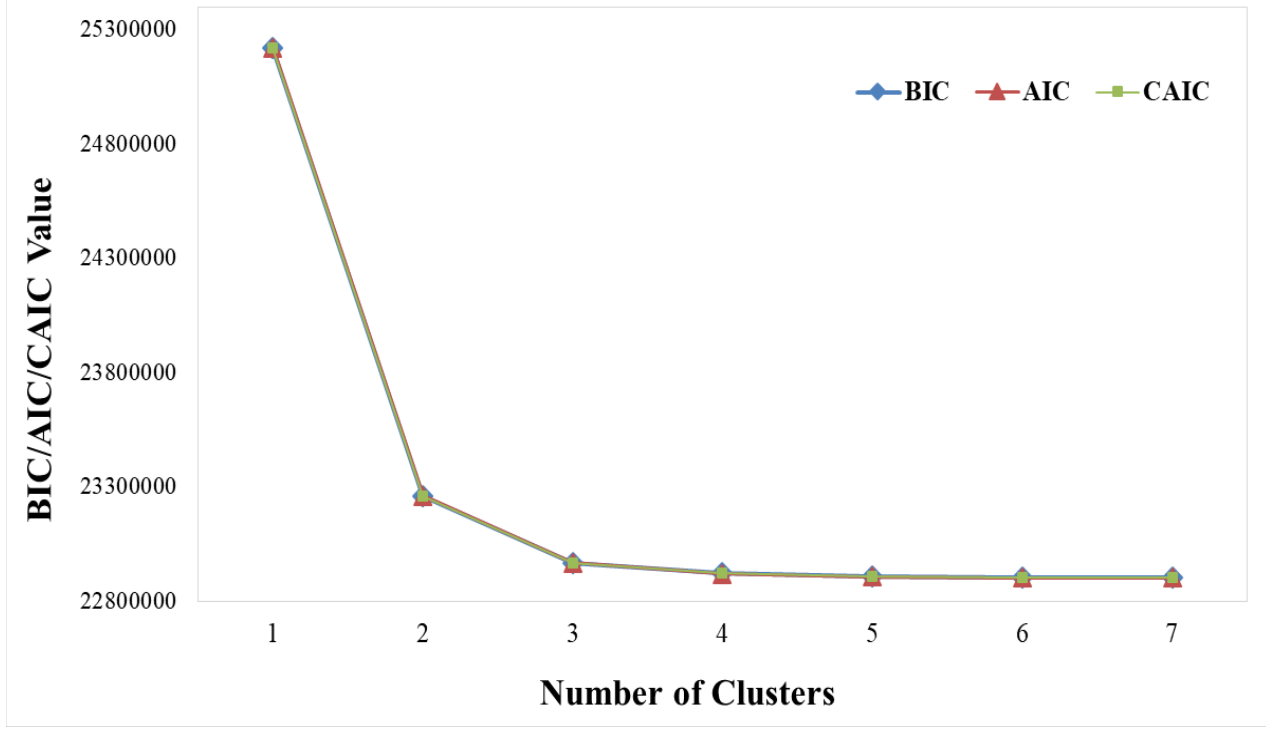

FIGURE 5 Identification of Number of Clusters for Vehicle Road Use Patterns

TABLE 5 Probabilities of Membership to Each Cluster

\begin{tabular}{llcccc}
\hline Parameters & Categories & cluster1 (\%) & cluster2 (\%) & cluster3 (\%) & cluster4 (\%) \\
\hline \multirow{3}{*}{ WO } & L1 & 0 & 4 & 40 & 95 \\
(workday)) & L2 & 1 & 18 & 45 & 0 \\
& L4 & 33 & 64 & 14 & 3 \\
& L1 & 66 & 14 & 1 & 2 \\
WE & L2 & 0 & 4 & 47 & 92 \\
(weekend) & L3 & 0 & 15 & 35 & 2 \\
& L4 & 20 & 71 & 18 & 6 \\
& L1 & 80 & 10 & 0 & 0 \\
HO & L2 & 1 & 11 & 51 & 0 \\
(holiday) & L3 & 2 & 18 & 28 & 47 \\
& L4 & 26 & 57 & 20 & 52 \\
\end{tabular}

12 (L1 to L4: vehicle traveling rates range from low to high)

13 
1 Cluster 1: $99 \%, 100 \%$ and $97 \%$ of the vehicles in this cluster have either the $3^{\text {rd }}$ or the $4^{\text {th }}$

2 level traveling rates on workdays, weekends and holidays. This cluster has high traveling 3 rate as $66 \%, 80 \%$ and $71 \%$ of the vehicles are categorized as the $4^{\text {th }} l e v e l$. We refer to this

4 cluster as "high-level road use pattern".

5 Cluster 2: $88 \%, 81 \%$ and $71 \%$ of the vehicles in this cluster have either the $3^{\text {rd }}$ or the $4^{\text {th }}$

6 level traveling rates on workdays, weekends and holidays. This cluster is different from

7 Cluster 1 as it only has $14 \%, 10 \%$ and $14 \%$ of the vehicles categorized as the $4^{\text {th }}$ level. This

8 cluster is referred to as "medium-high-level road use pattern".

9 Cluster 3: This cluster has $85 \%, 82 \%$ and $79 \%$ of the vehicles with a low level of traveling

10 rates (i.e. $1^{\text {st }}$ and $2^{\text {nd }}$ level) on workdays, weekends and holidays. We refer to this cluster as

11 "low-medium-level road use pattern".

12 Cluster 4: This cluster contains $95 \%$ and $92 \%$ of the vehicles that travelled at the $1^{\text {st }}$ level 13 on workdays and weekends, while $53 \%$ of the vehicles travelled at the $3^{\text {rd }}$ level or $4^{\text {th }}$ level 14 on holidays. This cluster is referred to as "low-level road use pattern".

TABLE 6 Characterizations for Each Cluster

\begin{tabular}{lll}
\hline Cluster & Characterization & Proportion (\%) \\
\hline 1: high-level road use pattern & $\begin{array}{l}\text { vehicles with a high level traveling rate on all the } \\
\text { types of days }\end{array}$ & 9 \\
2: medium-high-level road & $\begin{array}{l}\text { vehicles with a medium-high level traveling rate } \\
\text { on all the types of days }\end{array}$ & 35 \\
use pattern & $\begin{array}{l}\text { vehicles with a low-medium level traveling rate } \\
\text { on all the types of days }\end{array}$ & 52 \\
3: low-medium-level road & vehicles merely travel on holidays & 4 \\
use pattern & &
\end{tabular}

\section{Hourly Based Crash Frequency Analyses}

For the hourly based crash frequency analyses, REHP model and REHNB model were established. A total of four types of variables were found to be significant: roadway geometry characteristics, temporal features, traffic operational statuses and vehicle road use pattern proportions. Multicollinearities between variables were tested before estimating the model, and there was no correlation found between the variables.

The modeling results were shown in Table 7. From the model goodness-of-fit indexes, the REHNB model provided slightly better fitness. Therefore, the following section will only introduce the modeling results of REHNB model. 
TABLE 7 Modeling Results for REHP and REHNB Models

\begin{tabular}{|c|c|c|c|c|c|c|}
\hline \multirow[b]{2}{*}{ Variable } & \multicolumn{3}{|c|}{ REHP } & \multicolumn{3}{|c|}{ REHNB } \\
\hline & Coff. & s.d. & p value & Coff. & s.d. & p value \\
\hline \multicolumn{7}{|l|}{ Binary part } \\
\hline Intercept & -2.34 & 0.65 & $<0.001$ & -2.34 & 0.65 & $<0.001$ \\
\hline \multicolumn{7}{|c|}{ Roadway geometry characteristics } \\
\hline Ramp Type 2 & 0.70 & 0.21 & $<0.001$ & 0.70 & 0.21 & $<0.001$ \\
\hline Ramp Type 3 & 0.99 & 0.24 & $<0.0001$ & 0.99 & 0.24 & $<0.0001$ \\
\hline Ramp Type 4 & 1.15 & 0.17 & $<0.0001$ & 1.15 & 0.17 & $<0.0001$ \\
\hline \multicolumn{7}{|l|}{ Temporal features } \\
\hline Workday & -1.22 & 0.24 & $<0.0001$ & -1.22 & 0.24 & $<0.0001$ \\
\hline Morning Peak & 0.94 & 0.24 & 0.0001 & 0.94 & 0.24 & 0.0001 \\
\hline \multicolumn{7}{|l|}{ Traffic operational statuses } \\
\hline VMT & 0.10 & 0.03 & $<0.001$ & 0.10 & 0.03 & $<0.001$ \\
\hline Log Average Speed & -0.58 & 0.13 & $<0.0001$ & -0.58 & 0.13 & $<0.0001$ \\
\hline Shanghai Vehicles Proportion & -1.22 & 024 & $<0.0001$ & -1.22 & 024 & $<0.0001$ \\
\hline \multicolumn{7}{|c|}{ Vehicle road use pattern proportions } \\
\hline Low-Level Proportion & 59.94 & 1.90 & $<0.0001$ & 59.94 & 1.90 & $<0.0001$ \\
\hline Medium-High-Level Proportion & -7.10 & 0.78 & $<0.0001$ & -7.10 & 0.78 & $<0.0001$ \\
\hline \multicolumn{7}{|l|}{ Count part } \\
\hline Intercept & -3.60 & 1.04 & $<0.001$ & -4.25 & 1.18 & $<0.001$ \\
\hline \multicolumn{7}{|c|}{ Roadway geometry characteristics } \\
\hline Ramp Type 2 & 0.54 & 0.24 & $<0.05$ & 0.59 & 0.27 & $<0.05$ \\
\hline Ramp Type 3 & 0.65 & 0.26 & $<0.05$ & 0.71 & 0.29 & $<0.05$ \\
\hline Ramp Type 4 & 0.52 & 0.22 & $<0.05$ & 0.56 & 0.24 & $<0.05$ \\
\hline Log Length & 0.43 & 0.14 & $<0.05$ & 0.46 & 0.15 & $<0.05$ \\
\hline \multicolumn{7}{|l|}{ Traffic operational statuses } \\
\hline Shanghai Vehicles Proportion & -0.54 & 0.23 & $<0.001$ & -0.56 & 0.25 & $<0.001$ \\
\hline \multicolumn{7}{|c|}{ Vehicle road use pattern proportions } \\
\hline Low-Level Proportion & 13.19 & 3.53 & $<0.001$ & 14.25 & 4.11 & $<0.001$ \\
\hline Medium-High-Level Proportion & -2.67 & 1.33 & $<0.05$ & -2.92 & 1.56 & $<0.05$ \\
\hline Beta & - & & - & 1.44 & 0.91 & 0.1149 \\
\hline d1 & 0.44 & 0.08 & $<0.0001$ & 0.42 & 0.09 & $<0.0001$ \\
\hline $\mathrm{d} 2$ & 0.79 & 0.07 & $<0.0001$ & 0.79 & 0.07 & $<0.0001$ \\
\hline -2log-likelihood & & 116 & & & 116 & \\
\hline AIC & & 117 & & & 117 & \\
\hline BIC & & 117 & & & 117 & \\
\hline
\end{tabular}


3 Binary part as logistic model

4 The binary part of the REHNB model identifies the influencing factors for the possibility

5 of having crashes within the observation time period, where a positive regression

6 coefficient implies an increasing trend.

7 Roadway geometry characteristics

8 Ramp Type variable was found to be significant, for which Ramp Type 1 was selected as

9 the reference category. Compared to Ramp Type 1, Ramp Type 2, 3 and 4 are significant

10 with positive signs while Ramp Type 4 has the largest estimated coefficients. These indicate

11 the higher probability of crash occurrence on Ramp Type 2, 3 and 4 comparing to that on

12 Ramp Type 1. It implies that the off-on ramp type combination (Ramp Type 1) is the safest

13 among the other ramp types, while the on-on ramp type combination is among the most

14 dangerous.

\section{Temporal features}

16 For temporal features, Workday and Morning Peak variables were found to be significant.

17 The regression coefficient for Workday is negative, indicating that workdays are less likely

18 to incur hourly crashes as opposed to weekends and holidays. In terms of Morning Peak

19 variable, it has a positive sign, which implies that it is more likely to incur crashes during

20 morning peak hours.

\section{Traffic operational statuses}

22 With respect to traffic operational statuses, the VMT (vehicles miles traveled) variable was

23 found to be positive. Crashes have higher probabilities of occurrence as the VMT increases.

24 Average speed variable was included in the model with an exponential form, and found to

25 be significant with a negative sign. It is implied that the higher probability of crashes lies

26 in segments with lower average speed. Moreover, the proportion of vehicles with a

27 Shanghai license plate was found to be significant with a negative sign. It concluded that

28 roadway segments with a higher composition of local vehicles are expected to have a lower

29 possibility of crash occurrence.

30 Vehicle road use pattern proportions

31 In terms of vehicle road use pattern compositions, the regression parameter for Low-Level

32 Proportion variable is significant with a positive sign. It indicates that segments with a

33 larger proportion of vehicles with the low-level road use pattern are more likely to incur

34 crashes. On the contrary, the Medium-High-Level Proportion variable is negative, which

35 implies that the segments that have a larger proportion of vehicles with the medium-high-

36 level road use pattern are associated with less crashes.

39 As a counterpart to the binary part, the count part of the REHNB model reveals the 
1 impacting factors for the crash frequency, where positive regression coefficients indicate 2 the increasing number of crash occurrence.

3 Roadway geometry characteristics

4 For the roadway geometry characteristics, two variables were found to be significant:

5 Ramp Type and Log Length. The Ramp Type variables showed similar results as the binary

6 part. Ramp Type1 was used as the reference category, and the regression coefficients of

7 Ramp Type 2, 3 and 4 were all positive, where Ramp Type 3 had the largest estimated value.

8 It is indicated that segments with off-on ramp combination type (Ramp Type 1) have the

9 least number of crashes and segments with on-off ramp type combination (Ramp Type 3)

10 contain the most. It is consistent with the research performed by Yu et al. (20). Log Length

11 variable has a positive coefficient, which indicates that the number of crashes would

12 increase along with the length of roadway segment. A similar modeling result was found

13 in a study investigated by Chen (30).

14 Traffic operational statuses

15 Shanghai Vehicles Proportion variable was found to be significant with a negative sign,

16 which means that roadway segments with a higher composition of local vehicles are

17 expected to reduce the crash number.

18 Vehicle road use pattern proportions

19 With respect to vehicle road use pattern composition, similar to the binary part, Low-Level

20 Proportion variable and Medium-High-Level Proportion variable were found to be

21 significant. Low-Level Proportion variable is significantly positive, which indicates

22 segments that have a larger proportion of vehicles with the low-level road use pattern are

23 more likely to have more hourly crashes. Medium-High-Level Proportion variable is

24 negative, which implies that the segments that have a larger proportion of vehicles with the

25 medium-high-level road use pattern are associated with less crashes.

36 Several interesting findings are concluded: (i) a higher proportion of vehicles with the low-

37 level road use pattern on a roadway system would increase the possibility of crash

38 occurrence and the number of crashes. This type of vehicles is normally making leisure

39 trips and traveling from the suburban area or the outside of the city. Their unfamiliarity 
with the roadway and traffic conditions might have led to less experienced driving behaviors and unexpected hazardous driving scenarios, which further increase the crash

3 occurrence likelihood; (ii) A higher proportion of vehicles with the medium-high-level road

4 use pattern would reduce the crash likelihood. These vehicles might travel on the Shanghai urban expressway much more frequent and their drivers are familiar with the traffic dynamics, bottlenecks and road configurations which reduce the crash occurrence risk; (iii) A higher proportion of vehicles with Shanghai license plate was also found to reduce the crash likelihood and the number of crashes. It could also be explained by their familiarity with the infrastructures and traffic operational conditions of the roadway network.

Based on the modeling results, several recommendations are put forward. First, education, campaign and driver training would be the key to reduce traffic crashes during holidays and weekends targeting at drivers who make leisure trips to unfamiliar areas. Second, previous safety control and management measures were mostly based on the volume indicator (e.g. UK motorway control algorithm adopts similar variable-speed limit sign (VSLS) control strategy under the same volume level [43]). However, the proportions of vehicles making trips for different purposes (e.g. commuting, leisure, business) would vary significantly under the same volume level, which would lead to different degrees of crash occurrence risk. Therefore, implementing the measures (e.g. variable message signs) suitable for workdays may not be effective during weekends and holidays. Traffic control and management strategies aimed at different drivers should further be investigated. Furthermore, future studies could be conducted to understand the operational characteristics at vehicle-level (i.e. lane changing behavior, driving speed) to unveil the differences in driving behaviors between drivers who frequently use a roadway system and

Acknowledgements: This study was jointly sponsored by the Chinese National Natural Science Foundation (NSFC 71771174 and 71531011) and the 111 Project (B17032).

\section{Author contribution statement:}

34 The authors confirm contribution to the paper as follows: Study conception and design: $\mathrm{Yu}, \mathrm{R}$. and Wang, Y, data collection: Wang, Y. and Yu, R. analysis and interpretation of results: Yu, R., Wang, Y., Quddus, M. draft manuscript preparation: Yu, R., Wang, Y.,

37 Quddus, M, Li, Jian, Wang, X. All authors reviewed the results and approved the final version of the manuscript 
2 REFERENCE

3 [1] Treat, John R. Tri-level study of the causes of traffic accidents: an overview of final

4 results. Proceedings: American Association for Automotive Medicine Annual Conference. 51977.

6 [2] Guo, X., and C. Chen. Study of Traffic Characteristics and Vehicle Using Features

7 Based on Vehicle License Plate Data: A Case Study of Shanghai. China Transportation 8 Review, 2016.

9 [3] Li, D., T. Miwa, and T. Morikawa. Modeling Time-of-Day Car Use Behavior: A 10 Bayesian Network Approach. Transportation Research Part D: Transport and 11 Environment, 2016. 47: 54-66.

12 [4] Wan, X., J. Chen, W. Hu, and Y. Jing. Empirical Analysis of the Relationship between

13 Car Use Behavior and Urban Characteristics: Case Study of China.In 2008 International

14 Conference on Intelligent Computation Technology and Automation (ICICTA), 2008. 2: $15 \quad 358-363$.

16 [5] Wang, F., and D. Wang. Characteristics and determinants of car use in Beijing, Acta 17 Geographica Sinica-Chinese Edition, 2014. 69.6: 771.

18 [6] Chen, X., X. Yang, and Q. Shi. Estimation of Vehicle Usage Rate Based on Capture-

19 Recapture Model with License Plate Recognition Data. 11th International IEEE

20 Conference on Intelligent Transportation Systems, 2008.

21 [7] Lord, D., and F. Mannering. The Statistical Analysis of Crash-Frequency Data: A

22 Review and Assessment of Methodological Alternatives. Transportation Research Part A:

23 Policy and Practice, 2010. 44.5: 291-305.

24 [8] Coburn, T. C. Statistical and Econometric Methods for Transportation Data Analysis.

25 Technometrics, 2004. 46.4: 492-493.

26 [9] Chen, F., X. Ma, and S. Chen. Refined-Scale Panel Data Crash Rate Analysis Using

27 Random-Effects Tobit Model. Accident Analysis \& Prevention, 2014. 73: 323-332.

28 [10] Ma, X., F. Chen, and S. Chen. Modeling Crash Rates for a Mountainous Highway

29 Using Refined-Scale Panel Data.In Transportation Research Board Meeting, 2015.

30 [11] Berry, J.A., M., and G. S. Linoff. Data Mining Techniques: For Marketing, Sales, and 31 Customer Support. John Wiley \& Sons, Inc., 1997.

32 [12] Rai, P., and S. Shubha. A Survey of Clustering Techniques. International Journal of 33 Computer Applications, 2010. 7.12: 1-5.

34 [13] Fraley, C., and A. Raftery. Model-Based Clustering, Discriminant Analysis, and

35 Density Estimation. Journal of the American statistical Association, 2002. 97.458: 611$36 \quad 631$.

37 [14] Depaire, B., G. Wets, and K. Vanhoof. Traffic Accident Segmentation by Means of 38 Latent Class Clustering. Accident Analysis \& Prevention, 2008. 40.4: 1257-1266.

39 [15] Bock, H. H. Probabilistic Models in Cluster Analysis. Computational Statistics \& 
Data Analysis, 1996. 23.1: 5-28.

2 [16] Fraley, C., and A. E. Raftery. Model-Based Clustering, Discriminant Analysis, and

3 Density Estimation. Journal of the American Statistical Association, 2002. 97.458: 6114631.

5 [17] de Oña, J., G. López, R. Mujalli, and F. J. Calvo. Analysis of Traffic Accidents on

6 Rural Highways Using Latent Class Clustering and Bayesian Networks. Accident Analysis

7 \& Prevention, 2013. 51: 1-10.

8 [18] Sasidharan, L., K.-F. Wu, and M. Menendez. Exploring the Application of Latent Class

9 Cluster Analysis for Investigating Pedestrian Crash Injury Severities in Switzerland.

10 Accident Analysis \& Prevention, 2015: 219-228.

11 [19] Schreiber, J. B. Latent Class Analysis: An Example for Reporting Results. Research

12 in Social and Administrative Pharmacy, 2017. 13.6: 1196-1201.

13 [20] Yu, R., X. Wang, and M. Abdel-Aty. A Hybrid Latent Class Analysis Modeling

14 Approach to Analyze Urban Expressway Crash Risk. Accident Analysis \& Prevention, 15 2017. 101: 37-43.

16 [21] Lee, J., and F. Mannering. Impact of Roadside Features on the Frequency and Severity of Run-off-Roadway Accidents: an empirical analysis. Accident Analysis \& Prevention, 2002. 34.2: 149-161.

19 [22] Chin, H., and M. Quddus. Modeling Count Data with Excess Zeroes. Sociological methods \& research, 2003. 32.1: 90-116. [23] Shankar, V., J. Milton, and F. Mannering. Modeling Accident Frequencies as Zeroaltered Probability Processes: An Empirical Inquiry. Accident Analysis \& Prevention, 1997. 29.6: 829-837.

[24] Lord, D., S. P. Washington, and J. N. Ivan. Poisson, Poisson-Gamma and Zero-Inflated Regression Models of Motor Vehicle Crashes: Balancing Statistical Fit and Theory. Accident Analysis \& Prevention, 2005. 37.1: 35-46. [25] Lord, D., S. Washington, and J. N. Ivan. Further Notes on the Application of ZeroInflated Models in Highway Safety. Accident Analysis \& Prevention, 2007. 39.1: 53-57. [26] Hosseinpour, M., J. Prasetijo, A. S. Yahaya, and S. M. R. Ghadiri. A Comparative Study of Count Models: Application to Pedestrian-Vehicle Crashes Along Malaysia Federal Roads. Traffic Injury Prevention, 2013. 14.6: 630-638. Application to the Demand for Durable Goods. Econometrica, 1971. 39.5: 829-844. [28] Kassahun, W., T. Neyens, G. Molenberghs, C. Faes, and G. Verbeke. Marginalized Multilevel Hurdle and Zero-Inflated Models for Overdispersed and Correlated Count Data with Excess Zeros. Statistics in Medicine, 2014. 33.25: 4402-4419. [29] Son, H. D., Y.-J. Kweon, and B. B. Park. Development of Crash Prediction Models with Individual Vehicular Data. Transportation Research Part C: Emerging Technologies, 2011. 19.6: 1353-1363. 
1 [30] Chen, F., X. Ma, S. Chen, and L. Yang. Crash Frequency Analysis Using Hurdle

2 Models with Random Effects Considering Short-Term Panel Data. International Journal

3 of Environmental Research and Public Health, 2016. 13.11: 1043.

4 [31] Mannering, F. L., and C. R. Bhat. Analytic Methods in Accident Research:

5 Methodological Frontier and Future Directions. Analytic Methods in Accident Research,

6 2014. 1: 1-22.

7 [32] Aguero-Valverde, J. Full Bayes Poisson Gamma, Poisson Lognormal, and Zero

8 Inflated Random Effects Models: Comparing the Precision of Crash Frequency Estimates.

9 Accident Analysis \& Prevention, 2013. 50: 289-297.

10 [33] Chin, H. C., and M. A. Quddus. Applying the Random Effect Negative Binomial

11 Model to Examine Traffic Accident Occurrence at Signalized Intersections. Accident

12 Analysis \& Prevention, 2003. 35.2: 253-259.

13 [34] Chen, F., S. Chen, and X. Ma. Crash Frequency Modeling Using Real-Time

14 Environmental and Traffic Data and Unbalanced Panel Data Models. International Journal

15 of Environmental Research and Public Health, 2016. 13.6: 609.

16 [35] Min, Y., and A. Agresti. Random Effect Models for Repeated Measures of Zero-

17 Inflated Count Data. Statistical modelling, 2005. 5.1: 1-19.

18 [36] Yu, R., M. Abdel-Aty, and M. Ahmed. Bayesian Random Effect Models Incorporating

19 Real-Time Weather and Traffic Data to Investigate Mountainous Freeway Hazardous

20 Factors. Accident Analysis \& Prevention, 2013. 50: 371-376.

21 [37] Mo, B., R. li, and X. Zhan. Speed Profile Estimation Using License Plate Recognition

22 Data. Transportation research part C: emerging technologies, 2017. 82: 358-378.

23 [38] Magidson, J., and J. Vermunt. Latent Class Models for Clustering: A Comparison with

24 K-means. Canadian Journal of Marketing Research, 2001. 20.1: 36-43.

25 [39] Junior, JF Hair, et al. Multivariate data analysis with readings. London: Macmillan, 261992.

27 [40] B. Lewis, J., and D. A. Linzer. poLCA: An R Package for Polytomous Variable Latent 28 Class Analysis. Journal of statistical software, 2011. 42.10: 1-29.

29 [41] Wolfinger, Russell D. "Fitting Nonlinear Mixed Models with the New NLMIXED

30 Procedure." Proceedings of the 24th Annual SAS Users Group International Conference

31 (SUGI 24). 1999.

32 [42] Scheier, L. M., A. B. Abdallah, J. A. Inciardi, J. Copeland, and L. B. Cottler. Tri-city 33 Study of Ecstasy Use Problems: A latent Class Analysis. Drug and Alcohol Dependence, 34 2008. 98.3: 249-263.

35 [43] Allaby, P., B. Hellinga, and M. Bullock. Variable Speed Limits: Safety and Operational 36 Impacts of a Candidate Control Strategy for Freeway Applications. IEEE Transactions on 37 Intelligent Transportation Systems, 2007. 8.4: 671-680. 